\title{
Review of: "An intrinsic endothelial dysfunction causes cerebral small vessel disease"
}

\author{
Toshiki Mizuno ${ }^{1}$ \\ 1 Kyoto Prefectural University of Medicine
}

Potential competing interests: The author(s) declared that no potential competing interests exist.

In this research article, Quick et al. demonstrated that an intrinsic endothelial dysfunction leads to cerebral small vessel disease (CSVD) using normotensive ATP11b KO rats, which the authors identified. Dr. Takashi Koizumi (syupic@koto.kpu-m.ac.jp) helped me to review this article.

Thus, several points as indicated below need to be addressed by authors to improve the quality of the article.

\#1. Fig. 1B: It can be realized that the expression of ATP11b protein is decreased in the immunostaining image in KO, but it could not be confirmed that it disappears, considering the background. I would require more findings to support the results using Western blotting analysis.

\#2. Fig.2C and 2D: Expression changes in CLDN5 and ICAM-1 are shown in cultured cells. It is interesting how they are observed in brain sections. If you have additional data, Supplement figures would be added.

\#3. Page2, line 16-17: Authors described "Not all blood vessels are affected, with heterogeneity between vessels even within animals (Fig. $2 \mathrm{H}) "$. Were there any differences between regions such as the anterior circulation and the posterior circulation?

\#4. Fig. 4C: Please mention the modality of MRI at the legend (T2*-weighted sequences).

\#5. Fig. 4C: The resolution of microbleeds on the T2*-weighted images are too poor. To confirm microbleeds in the ATP11b KO model brain, the authors would be required to add supportive data; e.g. Hematoxylin and eosin stain.

\#6. Figure 4: Please clarify why the authors selected 3-4 months and 9-10 months groups on the MRI study, not the Juvenile (3-6 weeks old) and the Adult (24-30 weeks old) groups.

\#7 Suppl. Fig.4A: The notations of $\mathrm{i}$, ii, and iii on the corpus callosum are difficult to understand. Please 
correct. 